\section{Bone morphogenetic protein-4 and bone morphogenetic protein receptors expressions in the adult rat eye}

\author{
Yuka Maruyama-Koide, ${ }^{1,2}$ \\ Sumiko Mikawa, ${ }^{1}$ Takeshi Sasaki, ${ }^{1}$ \\ Kohji Sato ${ }^{1}$ \\ ${ }^{1}$ Department of Organ and Tissue \\ Anatomy, Hamamatsu University School \\ of Medicine, Hamamatsu, Shizuoka \\ ${ }^{2}$ Department of Ophthalmology, \\ Hamamatsu University School of \\ Medicine, Hamamatsu, Shizuoka, Japan
}

\begin{abstract}
We investigated the expressions of bone morphogenetic protein-4 (BMP4) and its receptors, bone morphogenetic protein receptor IA (BMPRIA), bone morphogenetic protein receptor IB (BMPRIB) and bone morphogenetic protein receptor II (BMPRII) in the adult rat eye. Interesting differences in expression profile were observed between BMPRIA and BMPRIB in the retina. BMPRIA-like immunoreactivity (IR) was very intensely seen in the photoreceptor layer, while BMPRIB-IR was mainly observed in the other layers. In the cornea, BMP4, BMPRIA, BMPRIB and BMPRII-IRs were abundantly seen in the cell body of basal cells in the corneal epithelium, and endothelium. In the lens, BMP4, BMPRIA, BMPRIB and BMPRIIIRs were observed in epithelial cells, lens cortical fiber cells, however they were not seen in the capsule and the central region of the lens. In the iris and ciliary body, strong BMP4 and BMPRIB-IRs were observed in nonpigmented epithelium. These results suggest that different kinds of BMP signaling should be needed in different areas in the adult eye to keep the shapes, differentiation levels, and functions of various cells.
\end{abstract}

\section{Introduction}

Bone morphogenetic protein-4 (BMP4) is a member of the transforming growth factor $\beta$ (TGF- $\beta$ ) superfamily. ${ }^{1}$ BMP4 signaling has been shown to play an important role in multiple biological events, including neural induction, ${ }^{2}$ tissue patterning, ${ }^{3}$ epithelial-mesenchymal interactions underlying organogenesis, ${ }^{4}$ lineage selection, ${ }^{5}$ and in the creation of stem cell 'niches' in developing and adult organs. ${ }^{6,7}$ BMP4 is synthe- sized as a large precursor, and subsequently cleaved to yield a carboxy-terminal mature protein. BMP4 exerts its biological functions by interacting with membrane-bound receptors belonging to the serine/threonine kinase family including bone morphogenetic protein receptor type I (BMPRIA, BMPRIB) and type II (BMPRII). ${ }^{8}$ These receptors form heteromeric complexes of type I and type II receptors in which type II is the ligand binding subunit which phosphorylates type I resulting in intracellular cascade events.

Also in the early stages of the developing eye, BMP4 has been reported to play pivotal roles, including the lens formation, topographic retinotectal projection, ${ }^{10}$ and apoptotic cell death. ${ }^{11}$ Although BMP4 and its receptors expressions have been well described in the early stages of the developing eye, ${ }^{9,12}$ little information is available for BMP4 and its receptors expressions in the adult eye. In the present study, we thus investigated the expression of BMP4 and its receptors, BMPRIA, BMPRIB and BMPRII in the adult eye. In the present study, we show that they are abundantly and differentially expressed in the adult eye.

\section{Materials and Methods}

\section{Animals}

Male Wistar rats at 7 weeks $(n=5)$ were used. For immunohistochemistry, five rats were perfused transcardially under deep anesthesia with saline followed by $0.1 \mathrm{M}$ phosphate buffer (PB) containing 4\% paraformaldehyde. Then eyes $(n=5)$ were removed rapidly, postfixed in the same fixative for $2 \mathrm{~h}$ at $4{ }^{\circ} \mathrm{C}$ and dehydrated in $100 \%$ ethanol. They were embedded in routinely paraffin wax, serially sectioned at $4 \mu \mathrm{m}$ and mounted on glass slides, and these sections were preserved at $-30^{\circ} \mathrm{C}$ until use to prevent the attenuation of immunoreactivity. ${ }^{13}$ All experiments conformed to the Guidelines for Animal Experimentation at Hamamatsu University School of Medicine on the ethical use of animals.

\section{Immunohistochemistry}

Paraffin sections were deparaffinized in xylene, dehydrated in decreasing alcohol solutions, and endogenous peroxidase was blocked with $0.3 \% \mathrm{H}_{2} \mathrm{O}_{2}$ in methanol for 20 min. For BMP4 staining, the sections were treated with $10 \%$ normal goat serum, $2 \%$ bovine serum albumin (BSA) and $0.2 \%$ Triton X-100 in $0.1 \mathrm{M}$ PB for $2 \mathrm{~h}$ at room temperature, and incubated further in mon-
Correspondence: Kohji Sato, Department of Organ and Tissue Anatomy, Hamamatsu University School of Medicine, 1-20-1 Handayama, Higashiku, Hamamatsu, Shizuoka 431-3192, Japan.

Tel. \& Fax: +81.53.435-2582.

E-mail: ksato@ hama-med.ac.jp

Key words: BMP4; BMPRIA; BMPRIB; BMPRII; eye

Contributions: YMK, SM, TS, KS, conception and design; YMK, SM, TS, immunostaining; YMK, SM, KS, immunostaining analysis; KS, TS, manuscript writing. All authors read and approved the final version of the manuscript.

Conflict of interest: the authors have no conflict of interest to disclose.

Funding: This work was supported by research grants from the Scientific Research Fund of the Ministry of Education, Science, and Cultures, Japan (No. 23591861 and 26462104) to TS.

Received for publication: 29 March 2017. Accepted for publication: 23 June 2017.

This work is licensed under a Creative Commons Attribution-NonCommercial 4.0 International License (CC BY-NC 4.0).

(C) Copyright Y. Maruyama-Koide et al., 2017 Licensee PAGEPress, Italy

European Journal of Histochemistry 2017; 61:2797 doi:10.4081/ejh.2017.2797

oclonal mouse anti-BMP4 (1:100 dilution; NCL-BMP-4, Novocastra, Newcastle, UK) overnight at $4{ }^{\circ} \mathrm{C}$. After washing with $0.1 \mathrm{M}$ $\mathrm{PB}$, sections were incubated in goat antimouse IgG with peroxidase complex (Histofine Simple Stain Rat MAX-PO(M); Nichirei, Tokyo, Japan) for $2 \mathrm{~h}$ at room temperature. For BMPRIA, BMPRIB and BMPRII staining, the sections were treated with $10 \%$ normal rabbit serum, $2 \%$ BSA and $0.2 \%$ Triton $\mathrm{X}-100$ in $0.1 \mathrm{M}$ PB for $2 \mathrm{~h}$ at room temperature, and incubated further in polyclonal goat anti-BMPRIA (1:50 dilution; sc-5676, Santa Cruz Biotechnology, Santa Cruz, CA, USA), goat anti- BMPRIB (1:100 dilution; sc-5679, Santa Cruz Biotechnology) and goat anti-BMPRII (1:50 dilution; sc-5683, Santa Cruz Biotechnology) for overnight at $4^{\circ} \mathrm{C}$, respectively. After washing with $0.1 \mathrm{M} \mathrm{PB}$, sections were incubated in rabbit anti-goat IgG with peroxidase complex (Histofine Simple Stain Rat MAX-PO(G); Nichirei) for $2 \mathrm{~h}$ at room temperature. After the final wash with $0.1 \mathrm{M} \mathrm{PB}$, immunoreaction was visualized with 3,3'-diaminobenzidine (DAB) (Wako, Osaka, Japan). The anti- 
BMP4 antibody was a mouse monoclonal antibody raised against the recombinant mouse bone morphogenetic protein-4. The anti-BMPRIA antibody and the antiBMPRIB antibody were affinity purified goat polyclonal antibodies raised against the N-terminal domains of each BMPR protein of human origin, respectively. The antiBMPRII antibody was an affinity purified goat polyclonal antibody raised against the cytoplasmic domain of BMPRII protein of human origin. For BMP4 pre-absorption controls, 1 nmol BMP4 recombinant protein was added to the diluted primary antibody $(100 \mu \mathrm{L})$, and incubated overnight at $4^{\circ} \mathrm{C}$. For BMPR1A, BMPRIB and BMPRII preabsorption controls, $40 \mu \mathrm{g}$ of each blocking peptide (sc-5676 P, sc-5679 P and sc-5683 P; Santa Cruz Biotechnology, respectively) which were linked with $20 \mu \mathrm{L}$ of NHS-activated Sepharose 4 Fast Flow (GE Healthcare Bio-Science KK, Tokyo, Japan), were added to the diluted primary antibodies $(100 \mu \mathrm{L})$, and incubated overnight at $4^{\circ} \mathrm{C}$. And then, each supernate was used for immunostaining. Three cross-sections of eyes at $80-\mu \mathrm{m}$ intervals were used for each immunostaining and observation per rat. Brightfield images were obtained with a microscope (Vanox-AHBS3; Olympus, Tokyo, Japan). They were further processed in a graphic editing program (Photoshop; Adobe, Tokyo, Japan) to obtain figures.

cDNA fragments encoding the C-terminal domain of rat BMP4 (aa 293-408, AY184241) were generated by RT-PCR using rat brain total RNA and subcloned into the pGEX-5X-1 vector. After transfection into BL21 E. coli, GST-BMP4 protein was expressed and purified with glutathione-sepharose 4B (Amersham Biosciences, Uppsala, Sweden) according to the manufacturer's instructions.

\section{Results}

\section{Specificity of anti-BMP4, BMPRIA, BMPRIB and BMPRII antibodies}

The specificity of antibodies used to detect BMP4, BMPRIA, BMPRIB and BMPRII proteins has been validated in our previous reports. ${ }^{14-17}$ Furthermore, to further confirm the specificity of these antibodies, we performed pre-absorption test in the retina and lens. In both regions, pre-absorption of the antisera with the corresponding antigens completely abolished the immunostaining. These data indicate that the antibodies specifically recognize BMP4 and $\mathrm{BMP}$ receptor proteins in the eye.

\section{Expressions of BMP4, BMPRIA,} BMPRIB and BMPRII in the retina

BMP4, BMPRIA, BMPRIB and BMPRII-IRs were observed in the adult rat retina (Figure 1 a-d). Pre-absorption of the
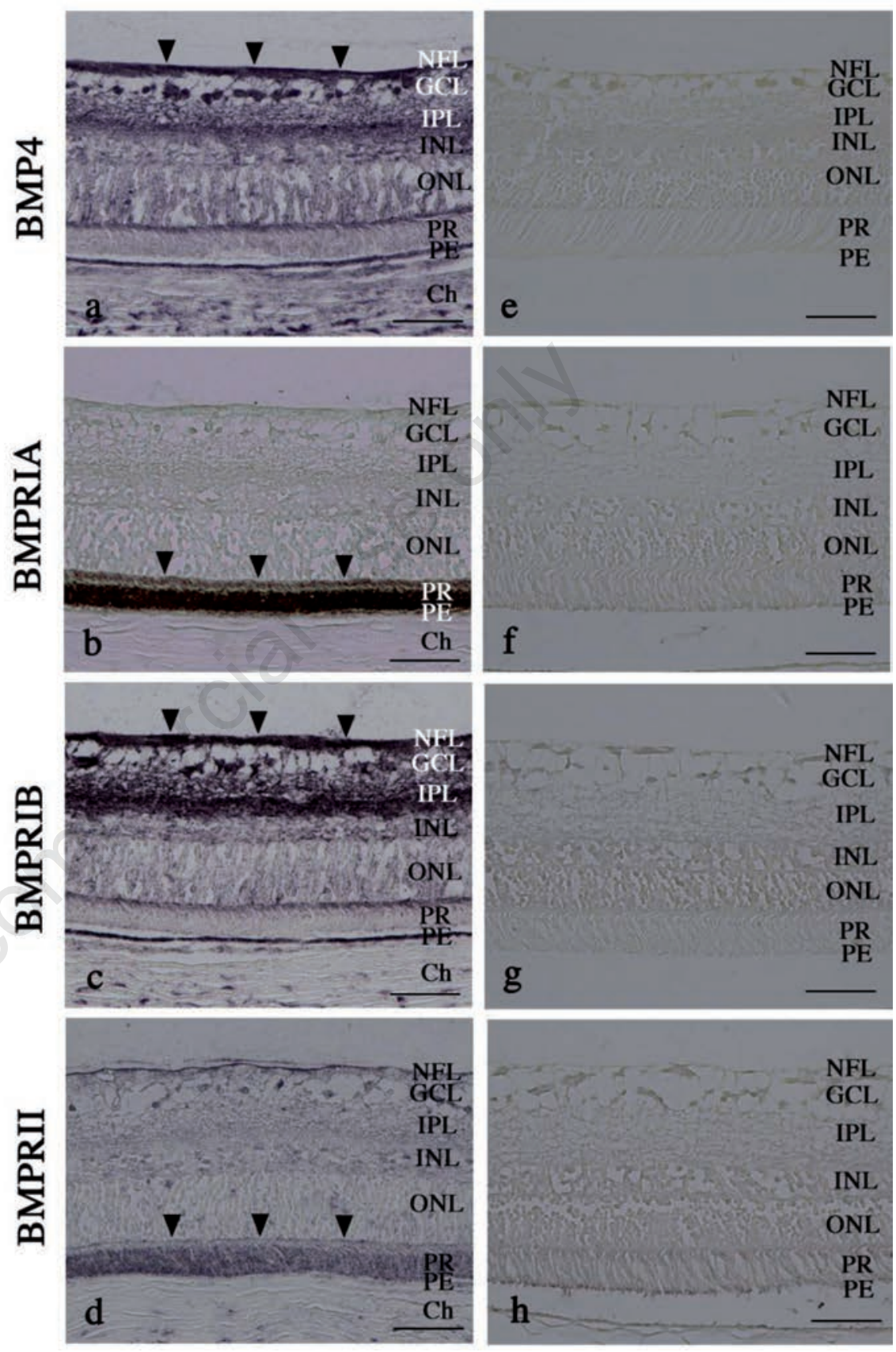

Figure 1. Pre-absorption tests for anti-BMP4 and BMP receptors antibody. BMP4 (a), BMPRIA (b), BMPRIB (c) and BMPRII (d) were immunostained with the corresponding antibodies in the adult rat retina. (e-h) Pre-absorption of the antibodies for BMP4, BMPRIA, BMPRIB and BMPRII using the corresponding antigens completely abolished the immunostainings, respectively. Note that intense BMP4-IR and BMPRIB-IR are observed in the nerve fiber layer and ganglion cell layer (arrowheads in a and c). In addition, note that, in the photoreceptor cell layer, intense BMPRIA-IR and BMPRII-IR are detected in the photoreceptor cell layer (arrowheads in b and d). Ch, choroidea; GCL, ganglion cell layer; IPL, inner plexiform layer; INL, inner nuclear layer; NFL, nerve fiber layer; ONL, outer nuclear layer; PE, pigmented epithelium; PR, Photoreceptor cell layer; Sc, sclera. Scale bars: $50 \mu \mathrm{m}$. 
Moderate BMP4-IR was seen in the inner plexiform layer, outer nuclear layer and pigmented epithelium (Figure 1a). BMP4 expression levels were weak in the inner nuclear layer and the outer segment of the photoreceptor cell layer (Figure 1a). Interesting differences in expression profile were observed between BMPRIA and BMPRIB in the retina. BMPRIA was very intensely expressed in the outer segment of the photoreceptor cell layer (arrowheads in Figure 1b), while BMPRIB expression was weak in this layer (Figure 1c). In contrast, intense BMPRIB expression was observed in the nerve fiber layer, ganglion cell layer, inner plexiform layer (arrowheads in Figure 1c), and pigmented epithelium, while BMPRIA expression in these areas were weak. BMPRII were intensely expressed in the outer segment of the photoreceptor cell layer (arrowheads in Figure 1d), and weakly expressed in the other layers (Figure 1d). In the choroidea, BMP4, BMPRIA, BMPRIB and BMPRII were weakly expressed (Figure 1 a-d). Closer observation further revealed their detailed expression profiles. In the nerve fiber layer, where axons of ganglion cells and astrocytes exist, strong BMP4 and BMPRIB-IRs were observed (arrowheads in Figure 2 a,c), while BMPRIA and BMPRII-IRs were weak (arrowheads in Figure 2 b,d). In the ganglion cell layer, ganglion cell bodies and their neurites were strongly stained with anti-BMP4 and anti-BMPRIB antibodies (arrowheads in Figure 2 a,c), while BMPRIA and BMPRII-IRs were weak (arrowheads in Figure 2 b,d). In the inner plexiform layer, where neurites from bipolar cells, amacrine cells and ganglion cells make synapses, many line-like structures were stained intensely with anti-BMPRIB antibody and moderately with anti-BMP4 antibody (Figure 2 a,c). In contrast, BMPRIA-IR and BMPRII-IR were weak (Figure $2 b$ and $d$ ). In the inner nuclear layers, where cell bodies of bipolar cells, amacrine cells and horizontal cells exist, BMP4, BMPRIA, BMPRIB and BMPRIIIRs were weakly observed around the cell bodies (Figure 2 a-d). Similarily, in the outer plexiform layer, where photoreceptor cells, bipolar cells and horizontal cells make synapses, BMP4, BMPRIA, BMPRIB and BMPRII-IRs were weakly observed (Figure $2 \mathrm{a}-\mathrm{d})$. In the outer nuclear cell layer, where cell bodies of photoreceptor cell exist, moderate BMP4 and BMPRIB-IRs and weak BMPRIA and BMPRII-IRs were seen around the cell bodies (Figure $2 \mathrm{e}-\mathrm{h}$ ). In the photoreceptor cell layer, the inner segment showed strong BMPRIA-IR, moderate BMP4 and BMPRIB-IRs and weak
BMPRII-IR (arrowheads in Figure 2 e-h). The outer segment showed very strong BMPRIA-IR, strong BMPRII-IR, and weak BMP4 and BMPRIB-IRs (arrowheads in
Figure 2 e-h). In the pigmented epithelium, strong BMPRIB-IR, moderate BMP4-IR, weak BMPRIA and BMPRII-IRs were observed (Figure 2 e-h). Relative intensities
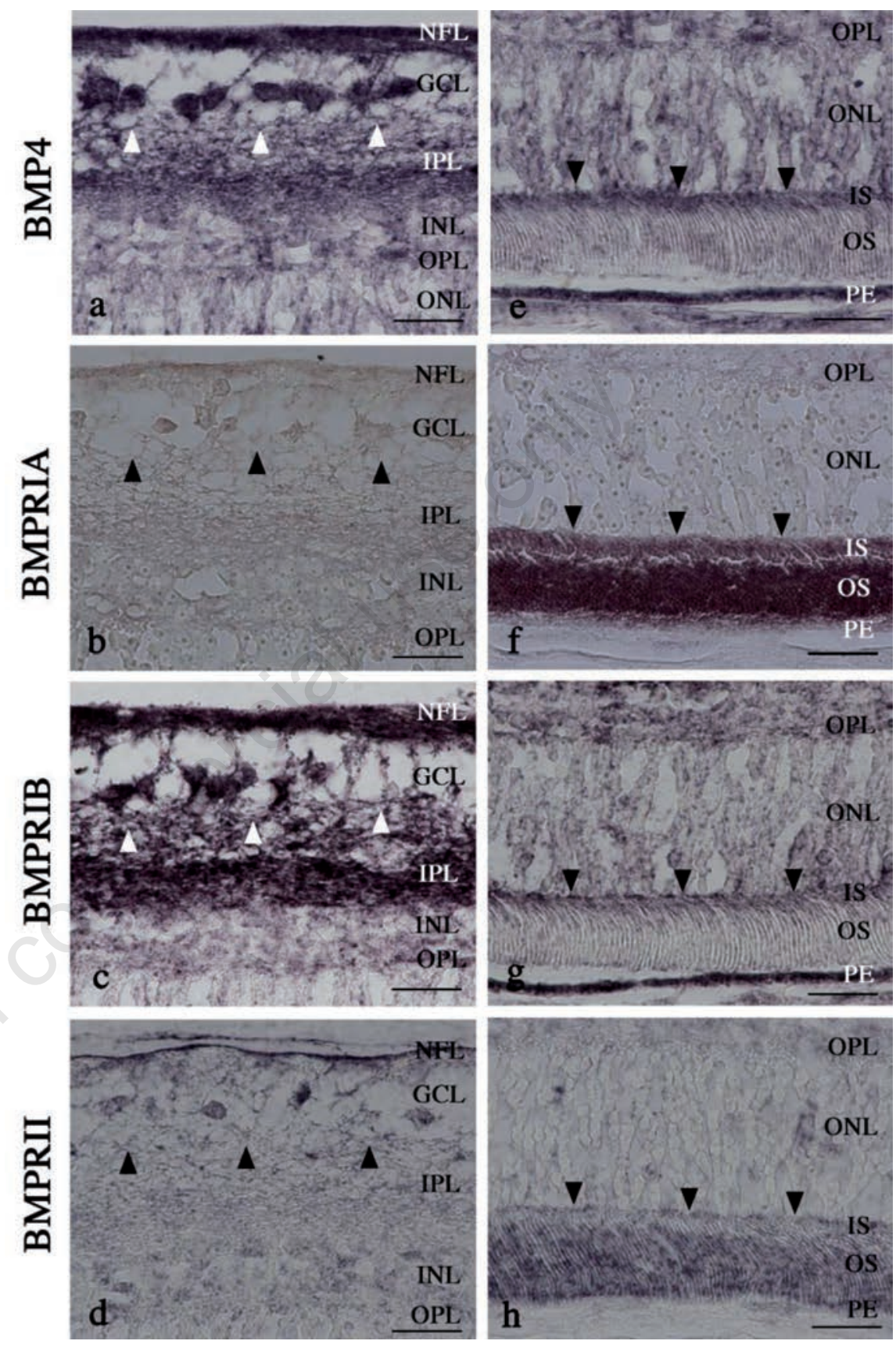

Figure 2. BMP4 and BMP receptors expressions in the adult rat retina. BMP4, BMPRIA, BMPRIB and BMPRII expressions in the upper layers (a-d, respectively) and lower layers (e-h, respectively) of the adult rat retina. Note that strong BMP4 and BMPRIB-IRs are observed in the nerve fiber layer and ganglion cell layer (arrowheads in a and c), while BMPRIA and BMPRII-IRs are weak there (arrowheads in $b$ and $d$ ). In addition, note that, in the photoreceptor cell layer, the inner segment shows strong BMPRIA-IR, moderate BMP4 and BMPRIB-IRs and weak BMPRII-IR (arrowheads e-h), and the outer segment exhibits very strong BMPRIA-IR, strong BMPRII-IR, and weak BMP4 and BMPRIB-IRs (arrowheads in e-h). Ch, choroidea; GCL, ganglion cell layer; IPL, inner plexiform layer; INL, inner nuclear layer; IS, inner segment of photoreceptor cell layer; NFL, nerve fiber layer; ONL, outer nuclear layer; OPL, outer plexiform layer; OS, outer segment of photoreceptor cell layer; PE, pigmented epithelium. Scale bar: $20 \mu \mathrm{m}$. 
of BMP4, BMPRIA, BMPRIB and BMPRII-like immunoreactivities (IRs) are summarized in Table 1.

\section{Expressions of BMP4, BMPRIA, BMPRIB and BMPRII in the cornea}

The cornea consists of the corneal epithelium, Bowman's membrane, stroma, and Descemet's membrane and endothelium. The corneal epithelium is stratified squamous epithelium with 4-5 layers. BMP4-IR was strongly observed in the cell bodies of basal cells in the corneal epithelium, while weakly seen in cells in the upper layers (arrowheads in Figure 3e). Similarly, BMPRIA, BMPRIB and BMPRII-IRs were also moderately observed in the cell bodies of basal cells (arrowheads in Figure 3f-h). In addition, weak BMP4, BMPRIA, BMPRIB and BMPRII expressions were observed in the endothelium (arrowheads in Figure 3a-d). BMP4, BMPRIA, BMPRIB and BMPRII-IRs were also weakly seen in the Bowman's membrane, stroma, and Descemet's membrane.

\section{Expressions of BMP4, BMPRIA, BMPRIB and BMPRII in the lens}

We observed intense BMP4, BMPRIA, BMPRIB and BMPRII-IRs in the lens (Figure 4 a-d). Pre-absorption of the antisera with the antigens for BMP4, BMPRIA, BMPRIB and BMPRII completely abolished these immunoreactivities (Figure $4 \mathrm{e}$ h). The mature lens is a polarized structure consisting of a mitotic epithelial layer that covers the anterior surface and terminally differentiated lens fibers that occupy the interior volume and the posterior surface. BMP4, BMPRIA, BMPRIB and BMPRIIIRs were moderately observed in epithelial cells (arrowheads in Figure 4 a-d). Interestingly strong BMP4, BMPRIA, BMPRIB and BMPRII-IRs were seen in lens cortical fiber cells (Figure 4 i-l). BMP4, BMPRIA, BMPRIB and BMPRIIIRs were strongly observed in the lateral edge of the equatorial region, and gradually decreased from the lateral edge to the center of the lens. Nuclei of lens cortical fiber cells were devoid of BMP4, BMPRIA, BMPRIB and BMPRII-IRs (arrowheads in Figure 4 i-1). They were not expressed in the capsule and the central region of the lens (Figure 4 i-1).

\section{Expressions of BMP4, BMPRIA, BMPRIB and BMPRII in the iris and ciliary body}

In the iris, strong BMP4 and BMPRIBIRs were observed in nonpigmented epithelium, while BMPRIA and BMPRII-IRs were weakly seen there (arrowheads in Figure 5 a-d). In the stroma, smooth muscles and pigmented epithelium in the iris, BMP4-IR was observed at moderate level, while BMPRIA, BMPRIB and BMPRIIIRs were observed at weak level (Figure 5 a-d). In the ciliary body, strong BMP4 and
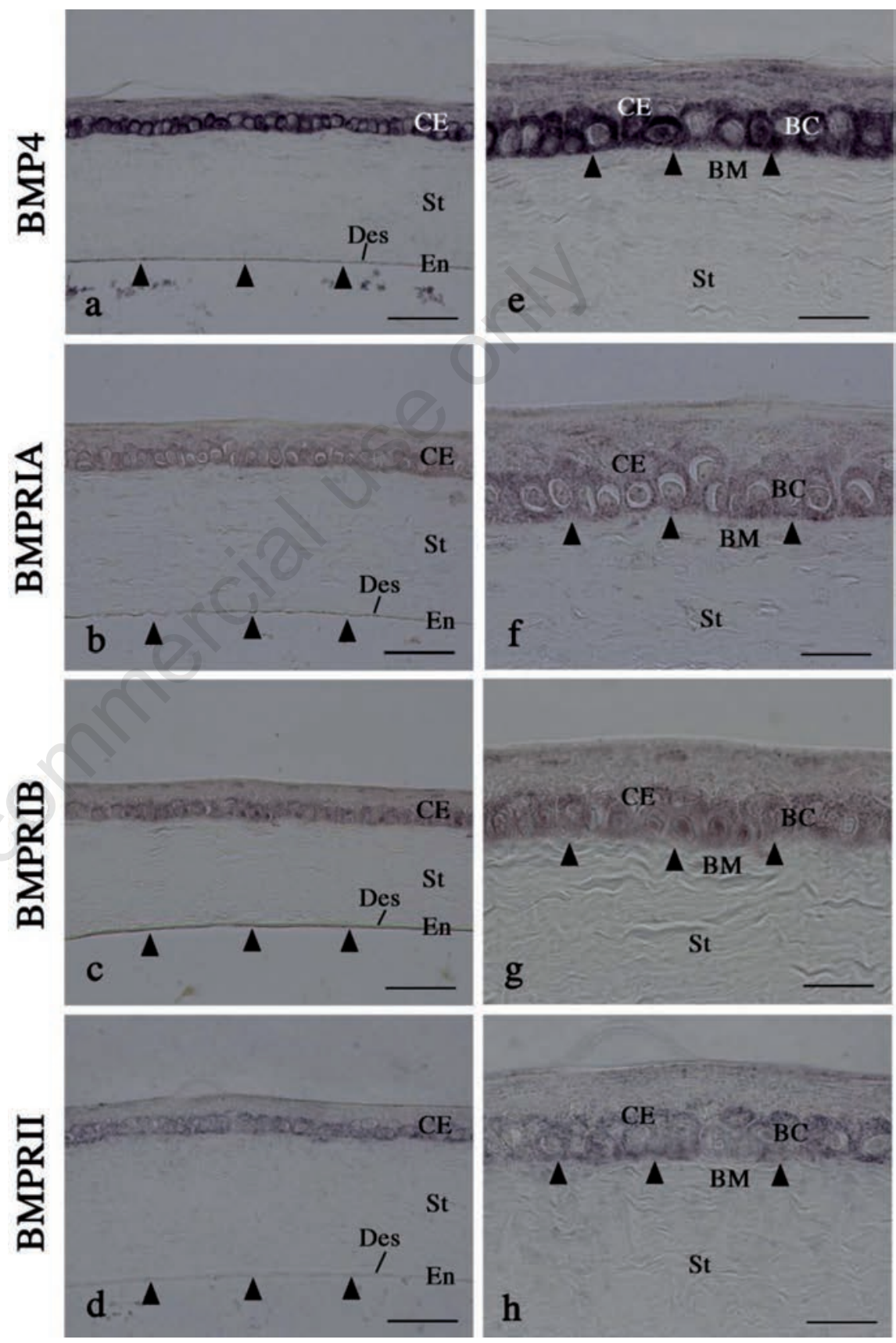

Figure 3. BMP4, BMPRIA, BMPRIB and BMPRII expressions in the adult rat cornea. Note that weak BMP4, BMPRIA, BMPRIB and BMPRII expressions are observed in the endothelium (arrowheads in a-d). In addition, note that strong BMP4-IR and moderate BMPRIA, BMPRIB and BMPRII-IRs are also moderately seen in the cell bodies of basal cells (arrowheads in f-h). BC, basal cells of corneal epithelium; BM, Bowman's membrane; CE, corneal epithelium; Des, Descemet's membrane; En, endothelium; St, stroma. Scale bars: $50 \mu \mathrm{m}$ (a-d); $20 \mu \mathrm{m}$ (e-h). 


\section{Discussion}

It is noteworthy that BMPRIA and BMPRIB were complementary expressed in the retina. BMPRIA was very intensely expressed in the photoreceptor cell layer, while BMPRIB was abundantly expressed in the other layers. Every photoreceptor cell has four regions: an outer segment, an inner segment, a cell body, and a synaptic terminal. The outer segment contains a stack of membranous disks, where light signals are converted into electronic signals. Our results indicate that, in a photoreceptor cell, BMPRIA expression was dominant in the outer segment and inner segment, while BMPRIB expression was dominant in the cell body and synaptic terminal, showing that BMPRIA and BMPRIB are differentially expressed in a single photoreceptor cell. Although the functional differences between BMPRIA and BMPRIB have not been clearly elucidated so far, we speculate that to keep the complicated structure of photoreceptor cells, different kinds of BMP signaling should be needed in different areas in photoreceptor cells.

Fischer et al. have reported that in the adult chick intraocular injections of BMP4 on the days immediately before a neurotoxic insult greatly reduced the amount of cell death induced by NMDA. ${ }^{18}$ Furthermore, in the rodent retina, the absence of BMPRIB results in elevated apoptotic cells in the inner nuclear layer, suggesting that BMP4mediated signaling is normally required to promote the survival of inner retinal neurons. ${ }^{19}$ Interestingly, in the present study, we observed BMP4, BMPRIB and BMPRII expressions in the inner nuclear layer, further supporting the possibility that BMP4 may act as a survival factor via BMPRIB and BMPRII in this region.

Table 1. Distribution and intensity of BMP4, BMPR1A, BMPR1B and BMPRII- IRs in the adult rat eye.

\begin{tabular}{|c|c|c|c|c|}
\hline Area & BMP4 & BMPR1A & BMPR1B & BMPRII \\
\hline \multicolumn{5}{|l|}{ Retina } \\
\hline Nerve fiber layer & +++ & + & +++ & + \\
\hline Ganglion cell layer & +++ & + & +++ & + \\
\hline Inner plexiform layer & ++ & + & +++ & + \\
\hline Inner nuculear layer & + & + & + & + \\
\hline Outer plexiform layer & + & + & + & + \\
\hline Outer nuclear layer & ++ & + & ++ & + \\
\hline \multicolumn{5}{|l|}{ Photoreceptor cell layer } \\
\hline inner segment & ++ & +++ & + & + \\
\hline outer segment & + & ++++ & + & +++ \\
\hline Pigmented epithelium & ++ & + & +++ & + \\
\hline Choroidea & + & + & + & + \\
\hline Sclera & + & + & + & + \\
\hline \multicolumn{5}{|l|}{ Cornea } \\
\hline Epithelium upper & + & + & + & + \\
\hline base & +++ & ++ & ++ & ++ \\
\hline Bowman's membrane & + & + & + & + \\
\hline Stroma & + & + & + & + \\
\hline Descemet's membrane & + & + & + & + \\
\hline Endothelium & + & + & + & + \\
\hline \multicolumn{5}{|l|}{ Lens } \\
\hline Subcapsular epithelium & ++ & ++ & ++ & ++ \\
\hline Lens fibers & +++ & +++ & +++ & +++ \\
\hline Lens capsule & n.d. & n.d. & n.d. & n.d. \\
\hline \multicolumn{5}{|l|}{ Iris } \\
\hline Stroma & + & + & + & + \\
\hline Pigmented epithelium & + & + & + & + \\
\hline Nonpigmented epithelium & +++ & + & +++ & + \\
\hline \multicolumn{5}{|l|}{ Ciliary body } \\
\hline Connective tissue & + & + & + & + \\
\hline Pigmented epithelium & + & + & + & + \\
\hline Nonpigmented epithelium & +++ & + & +++ & + \\
\hline
\end{tabular}

Relative intensities were estimated by visual comparison of immunostained slides: n.d., not detected; + , low; ++ , moderate; +++ , strong; ++++ , very strong.

We found that BMP4 was very intensely expressed in the cell bodies of basal cells in the corneal epithelium, while weakly expressed in cells in the upper layers (Figure 3e). Similarly, BMPRIA, BMPRIB and BMPRII were also abundantly expressed in the cell bodies of basal cells (Figure 3f-h). From these appearances, it is suggested that basal cells release $\mathrm{BMP} 4$, and $\mathrm{BMP} 4$ functions through BMPRIA, BMPRIB and BMPRII in autocrine or paracrine manners. We speculate that BMP4 signaling may be involved in the mechanism of basal cell differentiation.

BMP ligands and BMP receptors play critical roles in lens development. ${ }^{20}$. Faber et al. demonstrated that BMP signaling is required for development of primary lens fiber cells in the mouse. ${ }^{21}$ Noggin could suppress primary fiber cell elongation and mouse lens size in explant culture. ${ }^{21}$ When dominant negative BMPRIB was expressed in transgenic mice, the mice showed defects in the differentiation of primary lens fiber cells. ${ }^{21}$ In the present study, we observed abundant BMP4, BMPRIA, BMPRIB and BMPRII-IRs in epithelial cells and lens cortical fiber cells, indicating that BMP4 signaling is also important to regulate the differentiation processes of primary lens fiber cells even in the adult rat lens.

The aqueous humor is formed by the bilateral ciliary epithelium. The pigmented epithelium (PE) rests on the connective tissue stroma and the non-pigmented epithelium (NPE) is polarized with its basal lamina facing the posterior chamber of the eye. Interestingly, we found that strong BMP4 and BMPRIB expressions, and weak BMPRIA and BMPRII expressions in nonpigmented epithelium. Chang and colleagues showed that a heterozygous deficiency of BMP4 resulted in anterior segment dysgenesis, elevated intraocular pressure, and optic nerve abnormalies. ${ }^{22}$ Although the detailed mechanisms of BMP4 signaling in the adult ciliary body has been not elucidated, BMP4 signaling may be involved in regulating intraocular pressure conditions and be associated with the pathogenesis of glaucoma.

In this research, no quantitative analysis has been carried out, focusing on only qualitative analysis. Therefore, no conclusion about quantitative differences of BMPs and BMPRs was obtained from the results of this study, which is limitation of this research. In the future, quantitative investigation of BMPs and BMPRs in eyes will be needed. 

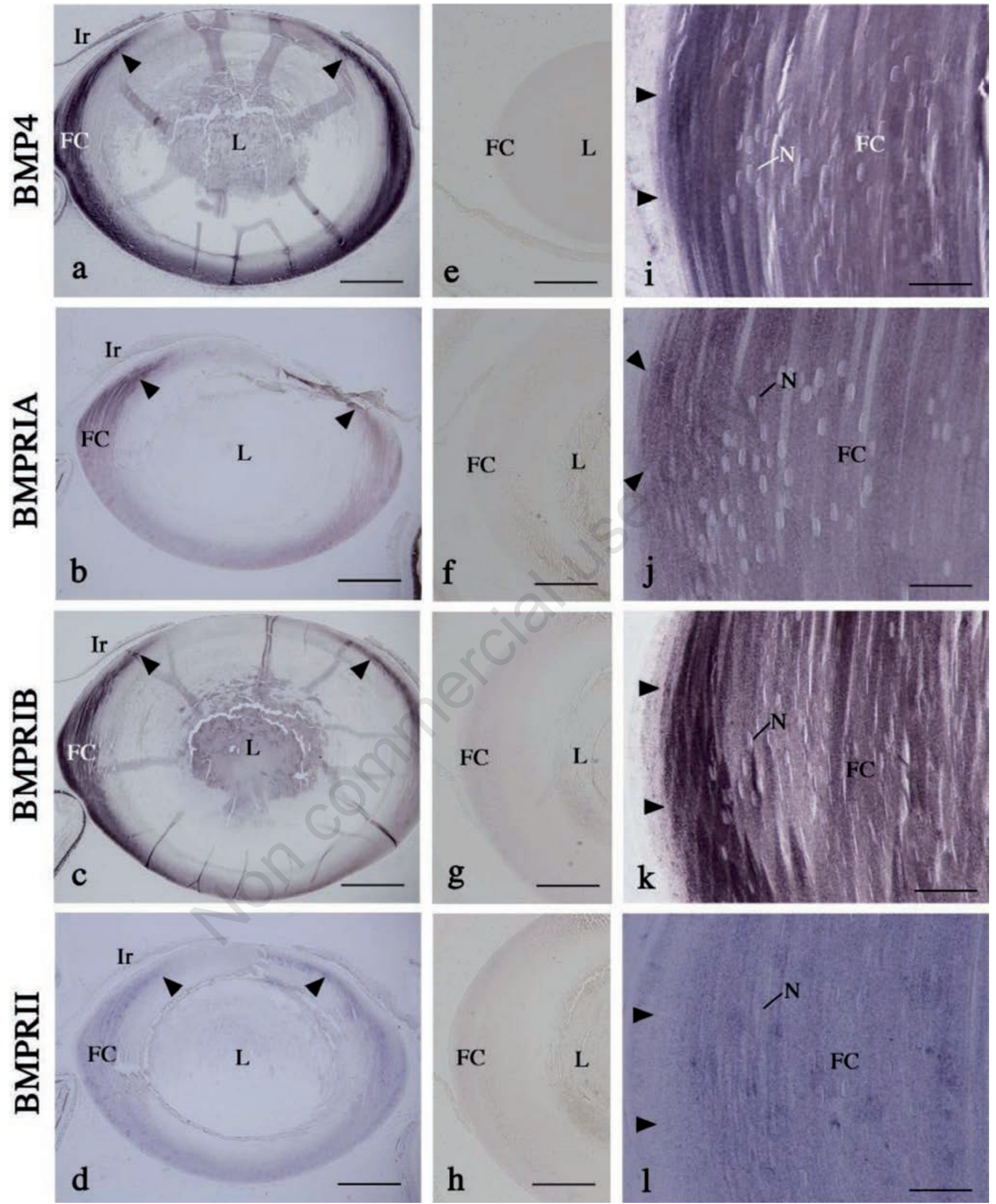

Figure 4. BMP4, BMPRIA, BMPRIB and BMPRII expressions in the adult rat lens. Intense BMP4, BMPRIA, BMPRIB and BMPRIIIRs are observed in the lens (a-d). Note that BMP4, BMPRIA, BMPRIB and BMPRII-IRs are moderately seen in epithelial cells (arrowheads in a-d). In addition, note that abundant BMP4, BMPRIA, BMPRIB and BMPRII expressions are also observed in lens fiber cells, while the nuclei of lens fiber cells are devoid of stainings (arrowheads in e-h). FC, fiber cells; Ir, iris; L, lens; N, nuclei of lens fiber cells. Scale bars: $0.5 \mathrm{~mm}(\mathrm{a}-\mathrm{d})$; and $20 \mu \mathrm{m}(\mathrm{e}-\mathrm{h})$. 

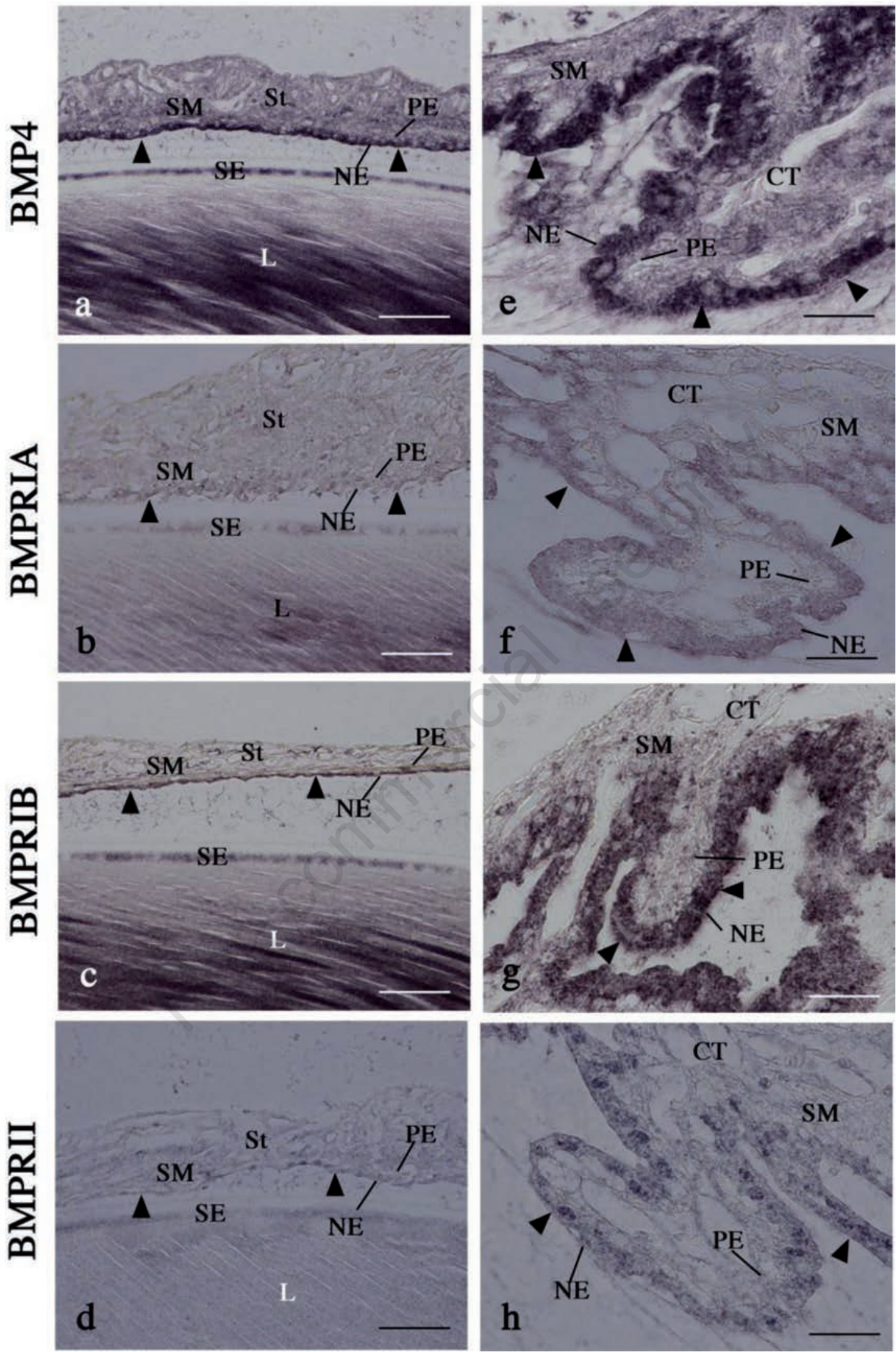

Figure 5. BMP4, BMPRIA, BMPRIB and BMPRII expressions in the adult rat iris and ciliary body. Note that strong BMP4 and BMPRIB-IRs are observed in the nonpigmented epithelium of the iris and ciliary body (arrowheads in a, $c$, e and $g$ ), while BMPRIA and BMPRII-IRs were weakly seen there (arrowheads in b, d, f, and h). CT; connective tissue; L, lens; NE, nonpigmented epithelium; PE, pigmented epithelium; SE, subcapsular epithelium; SM, smooth muscle, St, stroma. Scale bars: $20 \mu \mathrm{m}$. 


\section{References}

1. Hogan BL. Bone morphogenetic proteins in development. Curr Opin Genet Dev 1996;6:432-8.

2. Harland R. Neural induction. Curr Opin Genet Dev 2000;10:357-62.

3. McMahon JA, Takada S, Zimmerman LB, Fan CM, Harland RM, McMahon AP. Noggin-mediated antagonism of BMP signaling is required for growth and patterning of the neural tube and somite. Genes Dev 1998;12:1438-52.

4. Kulessa H, Turk G, Hogan BL. Inhibition of Bmp signaling affects growth and differentiation in the anagen hair follicle. EMBO J 2000;19:6664-74.

5. Mabie PC, Mehler MF, Kessler JA. Multiple roles of bone morphogenetic protein signaling in the regulation of cortical cell number and phenotype. J Neurosci 1999;19:7077-88.

6. Lim DA, Tramontin AD, Trevejo JM, Herrera DG, Garcia-Verdugo JM, Alvarez-Buylla A. Noggin antagonizes BMP signaling to create a niche for adult neurogenesis. Neuron 2000;28:713-26.

7. Watt FM, Hogan BL. Out of Eden: stem cells and their niches. Science 2000; 287:1427-30.

8. Sasai Y, De Robertis EM. Ectodermal patterning in vertebrate embryos. Dev Biol 1997;182:5-20.
9. Furuta Y, Hogan BL. BMP4 is essential for lens induction in the mouse embryo. Genes Dev. 1998;12:3764-75.

10. Sakuta H, Suzuki R, Takahashi H, Kato A, Shintani T, Iemura $\mathrm{Si}$, et al. Ventroptin: a BMP-4 antagonist expressed in a double-gradient pattern in the retina. Science 2001;293:111-5.

11. Trousse F, Esteve P, Bovolenta P. Bmp4 mediates apoptotic cell death in the developing chick eye. J Neurosci 2001; 21:1292-301.

12. Dudley AT, Robertson EJ. Overlapping expression domains of bone morphogenetic protein family members potentially account for limited tissue defects in BMP7 deficient embryos. Dev Dyn 1997;208:349-62.

13. Sasaki T, Kawabata Y, Suzuki N, Tanaka H, Sano M, Kato S, at al. Decreased D2-40 immunoreactivity in stored paraffin sections and methods for preserving it. Biotech Histochem 2014;89:412-8.

14. Maruyama Y, Mikawa S, Hotta Y, Sato $\mathrm{K}$. BMP4 expression in the developing rat retina. Brain Res 2006;1122:116-21.

15. Mikawa S, Wang C, Sato K. Bone morphogenetic protein- 4 expression in the adult rat brain. J Comp Neurol 2006; 499:613-25.

16. Miyagi M, Mikawa S, Hasegawa T, Kobayashi S, Matsuyama Y, Sato K. Bone morphogenetic protein receptor expressions in the adult rat brain. Neuroscience 2011;176:93-109.

17. Miyagi M, Mikawa S, Sato $T$, Hasegawa T, Kobayashi S, Matsuyama $\mathrm{Y}$, et al. BMP2, BMP4, noggin, BMPRIA, BMPRIB, and BMPRII are differentially expressed in the adult rat spinal cord. Neuroscience 2012;203:1226.

18. Fischer AJ, Schmidt M, Omar G, Reh TA. BMP4 and CNTF are neuroprotective and suppress damage-induced proliferation of Muller glia in the retina. Mol Cell Neurosci 2004;27:531-42.

19. Liu J, Wilson S, Reh T. BMP receptor $1 \mathrm{~b}$ is required for axon guidance and cell survival in the developing retina. Dev Biol 2003;256:34-47.

20. Wordinger RJ, Clark AF. Bone morphogenetic proteins and their receptors in the eye. Exp Biol Med 2007;232:97992.

21. Faber SC, Robinson ML, Makarenkova HP, Lang RA. Bmp signaling is required for development of primary lens fiber cells. Development 2002;129: 3727-37.

22. Chang B, Smith R, Peter M, Savinova O, Hawes N, Zabaleta A, et al. Haploinsufficient BMP-4 ocular phenotypes include anterior segment dysgenesis with elevated intraocular pressure. BMC Genet 2001;2:1. 